\title{
Understanding Mortality and Morbidity Meeting at Princess Marina Hospital: Case of Accident and Emergency Department (April 2014 to March 2015)
}

\author{
Article by Kabongo Leba \\ MBChB (UNILU), Accident and emergency department, Princess Marina Hospital, \\ M.Sc Clinical Research, Texila American University, Congo \\ Email: lebsbabs1984@gmail.com
}

\begin{abstract}
Background: The incidence and spectrum of mortality and morbidity in Botswana are not well established. Mortality data can be provided through the hospital records and documentation.

Objectives: Based on the importance given to accident and emergency at princess marina hospital by both the leaders and the entire population in Botswana, it was opportune to conduct this study. The major objective is to establish some epidemiological markers of death in our facility and therefore to provide factors surrounding death and solutions to reduce mortality.

Methods: A retrospective study has been conducted, based on monthly mortality and morbidity report provided by different doctors within the department.

Results: 27361 patients were seen during the said period with about 111 deaths (0.41\%). A total number of 10041(36.7 \%) patients were admitted to different wards for in-patients management. A couple of patients were brought in death after sudden collapse at home or being involved in road traffic accident. Roughly 9 patients died in accident and emergency each month with a mean of 2280 patients monthly attending the department. $56 \%$ of patient died with an internal medicine condition. $51 \%$ of died in accident and emergency were young adult on the range of $14-49$ year old. No significance value concerning the difference in gender.

Conclusion: The study provided details and a preliminary answer to cause of death grossly. Despite of comorbidities and the high number of HIV patients at the age ranging between 14 and 49 year old, having a high percentage of patients adults dying in accident and emergency is alarming and suggestions had been given to shrink this number.
\end{abstract}

Keywords: mortality, morbidity, mortality and morbidity, princess marina hospital, accident and emergency department, brought in dead, died in casualty,

\section{Introduction}

\section{Background}

The realization that errors play a significant role in mortality and morbidity in accident and emergency department together with poor triaging of the patients has generated interest and needs of quality improvement initiatives that took directly into human errors.

After initially updating the triage tool in 2009 and implementing it in 2010, and In order to provide high quality care, medical staff members in accident and emergency department at princess marina hospital engage quarterly, if possible, in an objective, non-judgmental review of adverse events and outcomes happened during the management of some selective patients .

Generally, the mortality and morbidity meeting will give a statistical report followed by a clear discussion concerning some selected cases of patients who died after being seen in the department or during their stay in the department. 
Texila International Journal of Medicine

Volume 4, Issue 2, Dec 2016

The choice of these cases depends upon several criteria including, but not limited to, presenter's judgment, poor management (delayed attendance, treatment given, protocols followed...), lessons learned and improvement for the future.

\section{Definition of concept}

- The free Dictionary defines "mortality" as the quality and condition of being mortal. "Mortal" is considered to be a group like "human race".

- Morbidity is a term used to describe how often a disease occurs in a specific area or is a term used to describe a focus on death.

- Mortality and morbidity meeting is a conference where regularly the staff take an indepth, franc and opened analysis of some incidents which happened during the management of some patients leading to death or near death; with the purpose of learning and improvement.

\section{Objective of mortality and morbidity ( $M$ and $M$ )}

Mortality and morbidity meeting is one of the key component of workplace-based learning, providing an opportunity to the medical staff in accident and emergency to discuss errors and adverse events which occur in the department during a certain period of time. The discussion is always done in a very friendly and open manner.

During the meeting, the staff will answer to some specific questions:

- What happened?

- Why did it occur?

- How could the issue have been prevented or better managed?

- What are the key learning points?

\section{Methodology}

This is a retrospective study. An archival data collection and archival compilation were done to collect all the data for this report. Generally a Medical officer in accident and emergency in collaboration with Healthcare auxiliaries' staff conduct the data collection under the supervision of the medical officer.

Some tools will be used to collect data:

- Departmental admission book: this book recorded all the patients seen in the department.

- "Died in Accident and emergency" book: this book records all the patients died while in the department.

- "Brought in dead" book: for all death before arrival to the department.

- Nursing mortality and morbidity statistics

- Accident and emergency triage form: containing nurses and doctors 'notes.

- Medical record information store room.

After collecting all the data, the medical staff assigned for the M\&M at a specific period compiles all the data.

After selecting cases for discussions, the concerned officer goes through the Notes (doctors and nurses) as well as outpatient cards if available and admitting department if reported.

During this report, we will give a statistical report of all the patients seen and died in Accident and emergency during the specific of time given. This will be followed by the presentation of some interesting case which was discussed by the department.

A discussion will be conducted generating conclusion and recommendation for the future at the end of the report.

\section{Results and discussion}

\section{Presentation of the department}

- Princess marina hospital is the biggest referral hospital with nearly 500 beds. It situated in the center of Gaborone, the capital city of Botswana. It is a specialized hospital running a multidisciplinary department, as teaching and tertiary hospital. 
- The accident and emergency department is one of the units of the Hospital. It is the major entrance to the hospital. It is situated by the entrance of the hospital with an emergency gate, opposite to the transport office for ambulances. The accident and emergency department is divided into different areas including

1. Head of department office:

2. Triage office:

3. Patient Waiting area

4. Family waiting area

5. Bays(1 to 10$)$ and corridors(1 to 7 )

6. Resuscitation room

7. Side laboratory

8. Kitchen

9. Sister in charge office

10. Store room

11. Disaster room

12. Doctors station

13. Nurse's station.

14. Isolation room.

- At the time this data were collected, the medical staff comprises the head of department, one emergency specialist under University of Botswana, 10 medical officers, 4 residents, and 2 visiting residents.

- The nurse's staff comprises the matron and sister in charge of the unit, 15 nurses, and 7 health care auxiliaries.

- A private company provides pottering services and cleaning services.

- The accident and emergency department only admits to the ward or discharge to the referring hospital or home. The department does not contain an observation ward.

- The Accident and emergency department has the mandate to manage and stabilize efficiently and effectively trauma and critically ill patients.

\section{Presentation of the results and interpretation}

Table 1. Summary of patients attended in Accident and emergency (A/E) from 01.04.2014 to 31.03.2015

\begin{tabular}{|l|l|l|l|l|}
\hline & Died in A/E & BID & Admission & Total \\
\hline April14 & 8 & 27 & 804 & 2210 \\
\hline May14 & 13 & 22 & 757 & 2136 \\
\hline June14 & 9 & 17 & 757 & 2035 \\
\hline July14 & 6 & 30 & 820 & 2301 \\
\hline august14 & 18 & 28 & 855 & 2318 \\
\hline September14 & 8 & 25 & 889 & 2419 \\
\hline October14 & 7 & 13 & 833 & 2440 \\
\hline November14 & 6 & 17 & 823 & 2277 \\
\hline December14 & 10 & 11 & 752 & 1966 \\
\hline January15 & 7 & 8 & 732 & 2083 \\
\hline February15 & 7 & 8 & 763 & 2574 \\
\hline March15 & 12 & 11 & 1256 & 2602 \\
\hline Total & 111 & 214 & 10041 & 27361 \\
\hline percentage & 0.41 & 0.78 & 36.7 & 100 \\
\hline
\end{tabular}

The table above represents the summary of all the patients seen and recorded according to their outcomes. For the period of April 2014 to march 2015, 27361 patients seek medical care in accident and emergency department at our facility. $36.7 \%$ were admitted, while $0.41 \%$ died within the department. We still have quite a high number of brought in dead cases, despite of different information given to the public. 
Texila International Journal of Medicine

Volume 4, Issue 2, Dec 2016

Table 2. Summary of patient died in Accident and Emergency (A/E) per month

Table 2.1. patients died in A/E during april2014

\begin{tabular}{|l|l|l|l|l|}
\hline Month & Disease & Gender & Age & $\begin{array}{l}\text { Source of } \\
\text { referral }\end{array}$ \\
\hline & $\begin{array}{l}\text { Cancer breast } \\
\text { with } \\
\text { hyperglycemia }\end{array}$ & F & 55 & Local clinic \\
\hline & $\begin{array}{l}\text { Per } \\
\text { Vaginalbleeding? } \\
\text { malignancy }\end{array}$ & F & 85 & Local clinic \\
\hline APRIL & $\begin{array}{l}\text { Traditional } \\
\text { medicine } \\
\text { intoxication }\end{array}$ & M & 47 & EMS \\
\hline & PCP & F & 50 & EMS \\
\hline & $\begin{array}{l}\text { Hepatomegaly } \\
\text { ?cause }\end{array}$ & F & 60 & Local clinic \\
& $\begin{array}{l}\text { Intestinal } \\
\text { obstruction }\end{array}$ & F & 40 & $\begin{array}{l}\text { District } \\
\text { hospital }\end{array}$ \\
\hline & HI/RTA & F & 31 & EMS \\
\hline & AIDS & F & 33 & Local clinic \\
\hline
\end{tabular}

During April 2014, 8 patients died while still in the unit. More than half of them were on the range of 14-49 years old and only one male patient among all.

Table 2.2. patients died in A/E in May 2014

\begin{tabular}{|c|c|c|c|c|}
\hline \multirow[t]{6}{*}{ Month } & Disease & Gender & Age & $\begin{array}{l}\text { Source of } \\
\text { referral }\end{array}$ \\
\hline & PTB/Renal failure & $\mathrm{M}$ & 35 & $\begin{array}{l}\text { District } \\
\text { hospital }\end{array}$ \\
\hline & $\begin{array}{l}\text { Advanced breast } \\
\text { Cancer }\end{array}$ & F & 33 & EMS \\
\hline & Collapse ?cause & $\mathrm{F}$ & 33 & EMS \\
\hline & RTA/Polytrauma & $\mathrm{M}$ & 32 & local hospital \\
\hline & Hematuria?cause & $\mathrm{M}$ & 72 & Local clinic \\
\hline \multirow[t]{8}{*}{ May14 } & $\begin{array}{l}\text { Hepatorenal } \\
\text { failure with } \\
\text { electrolyte } \\
\text { imbalance }\end{array}$ & $\mathrm{F}$ & 82 & Local clinic \\
\hline & PTB & $\mathrm{F}$ & 55 & EMS \\
\hline & $\begin{array}{l}\text { Acute respiratory } \\
\text { distress?cause }\end{array}$ & F & 39 & Local clinic \\
\hline & PTB & $\mathrm{M}$ & 43 & EMS \\
\hline & $\begin{array}{l}\text { Hepatorenal } \\
\text { syndrome }\end{array}$ & $\mathrm{F}$ & 37 & $\begin{array}{l}\text { Local } \\
\text { hospital }\end{array}$ \\
\hline & $\begin{array}{l}\text { Aspiration } \\
\text { pneumonia }\end{array}$ & $\mathrm{M}^{-}$ & 3 & $\begin{array}{l}\text { Local } \\
\text { hospital }\end{array}$ \\
\hline & $\begin{array}{l}\text { Renal failure/HIV } \\
\text { encephalitis }\end{array}$ & M & 32 & Local clinic \\
\hline & $\begin{array}{l}\text { Stevens } \\
\text { Johnson's } \\
\text { syndrome }\end{array}$ & $\mathrm{M}$ & 39 & Local clinic \\
\hline
\end{tabular}


13 patients died in May 2014 with a pediatric patient who came from a referring hospital. Mostly Female patients died during the course of this month. About $77 \%$ were young adult. Among them only 1 has a road traffic accident. The remaining has some medical condition.

Table 2.3. patients died in A/e in June 2014

\begin{tabular}{|l|l|l|l|l|}
\hline Month & Disease & Gender & Age & $\begin{array}{l}\text { Source of } \\
\text { referral }\end{array}$ \\
\hline & $\begin{array}{l}\text { Hemoperitoneum } \\
\text { post blunt } \\
\text { abdominal trauma }\end{array}$ & M & 32 & $\begin{array}{l}\text { Local } \\
\text { hospital }\end{array}$ \\
\hline $\begin{array}{l}\text { Severe } \\
\text { pneumonia/PCP }\end{array}$ & F & 40 & Local clinic \\
\hline & $\begin{array}{l}\text { Severe pneumonia } \\
\text { with CCF } \\
\text { exacerbation }\end{array}$ & F & 43 & Local clinic \\
& $\begin{array}{l}\text { Severe } \\
\text { pneumonia/septic } \\
\text { shock }\end{array}$ & F & 48 & Self-referral \\
\hline & Severe RTI & F & 59 & EMS \\
\hline & $\begin{array}{l}\text { Pleural effusion } \\
\text { Aspiration }\end{array}$ & F & 34 & $\begin{array}{l}\text { Local } \\
\text { hospital }\end{array}$ \\
\hline & Fneumonia & F & 29 & Local clinic \\
\hline & PTB & F & 31 & Local clinic \\
\hline
\end{tabular}

9 deaths were recorded in June. All were adult with a $78 \%$ of female and $22 \%$ male. Except the old lady with severe RTI all the remaining are ranging between 14-49 years old.

Table 2.4. patients died in A/E in July 2014

\begin{tabular}{|l|l|l|l|l|}
\hline & Diseases & Gender & Age & $\begin{array}{l}\text { Source of } \\
\text { referral }\end{array}$ \\
\hline & PTB & F & 33 & Local clinic \\
\hline & $\begin{array}{l}\text { Hepatic } \\
\text { encephalopathy }\end{array}$ & $\mathrm{F}$ & 38 & Local clinic \\
\hline July & $\begin{array}{l}\text { Subdural } \\
\text { hemorrhage }\end{array}$ & $\mathrm{M}$ & 42 & $\begin{array}{l}\text { Local } \\
\text { hospital }\end{array}$ \\
\hline & CVA & $\mathrm{F}$ & 70 & EMS \\
\hline & AIDS & $\mathrm{M}$ & 53 & EMS \\
\hline & $\begin{array}{l}\text { Ruptured } \\
\text { aneurysm }\end{array}$ & $\mathrm{F}$ & 54 & $\begin{array}{l}\text { Local } \\
\text { hospital }\end{array}$ \\
\hline
\end{tabular}

6 patients recorded dead. Oneunusual diagnosis of ruptured aneurysm had been reported as well. 2 third are female.

Table 2.5. patients died in A/E in august 2014

\begin{tabular}{|l|l|l|l|l|}
\hline & Diseases & Gender & Age & $\begin{array}{l}\text { Source of } \\
\text { referral }\end{array}$ \\
\hline & Renal failure & $\mathrm{M}$ & 55 & Self \\
\hline & $\begin{array}{l}\text { Severe } \\
\text { Gastroenteritis }\end{array}$ & $\mathrm{F}$ & 5 months & Local clinic \\
\hline & $\begin{array}{l}\text { Severe } \\
\text { pneumonia }\end{array}$ & $\mathrm{M}$ & 76 & EMS \\
\hline & Urosepsis & $\mathrm{M}$ & 53 & EMS \\
\hline
\end{tabular}


Texila International Journal of Medicine

Volume 4, Issue 2, Dec 2016

\begin{tabular}{|c|c|c|c|c|}
\hline & PTB & $\mathrm{F}$ & 32 & Local clinic \\
\hline & $\begin{array}{l}\text { Anemia with } \\
\text { CCF }\end{array}$ & $\mathrm{M}$ & 81 & Local clinic \\
\hline & $\begin{array}{l}\text { Severe } \\
\text { pneumonia }\end{array}$ & Male & 37 & EMS \\
\hline \multirow[t]{11}{*}{ august } & $\begin{array}{l}\text { Severe } \\
\text { malnutrition }\end{array}$ & $\mathrm{M}$ & 10months & Local clinic \\
\hline & $\begin{array}{l}\text { Crypto } \\
\text { meningitis }\end{array}$ & $\mathrm{M}$ & 17 & Local clinic \\
\hline & $\begin{array}{l}\text { Incarcerated } \\
\text { hernia }\end{array}$ & $\mathrm{M}$ & 70 & Local clinic \\
\hline & $\begin{array}{l}\text { Polytrauma post } \\
\text { RTA }\end{array}$ & $\mathrm{M}$ & 53 & EMS \\
\hline & $\begin{array}{l}\text { Crypto } \\
\text { meningitis }\end{array}$ & 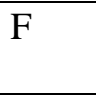 & 48 & EMS \\
\hline & CVA & $\mathrm{M}$ & 57 & $\begin{array}{l}\text { Local } \\
\text { hospital }\end{array}$ \\
\hline & $\begin{array}{l}\text { Massive } \\
\text { intracranial } \\
\text { hemorrhage }\end{array}$ & $\mathrm{M}$ & 36 & Local clinic \\
\hline & Pneumonia & $\mathrm{M}$ & 36 & $\begin{array}{l}\text { Local } \\
\text { hospital }\end{array}$ \\
\hline & CCF & $\mathrm{M}$ & 80 & Local clinic \\
\hline & $\begin{array}{l}\text { Head injury } \\
\text { post assault }\end{array}$ & $\mathrm{F}$ & 80 & $\begin{array}{l}\text { Local } \\
\text { hospital }\end{array}$ \\
\hline & CRF & $\mathrm{M}$ & 50 & Local clinic \\
\hline
\end{tabular}

The highest number of death. 2 infants died in a/e during this period. There was a very unusually assault; an 80 years old female who died with head injury. $78 \%$ of deaths were male. About $60 \%$ deaths beyond 50 years old.

Table 2.6. patients died in A/E in September 2014

\begin{tabular}{|c|c|c|c|c|}
\hline & Diseases & Gender & Age & $\begin{array}{l}\text { Source of } \\
\text { referral }\end{array}$ \\
\hline & $\begin{array}{l}\text { Electrolyte } \\
\text { imbalance with } \\
\text { renal failure }\end{array}$ & $\mathrm{F}$ & 46 & $\begin{array}{l}\text { Local } \\
\text { hospital }\end{array}$ \\
\hline & $\begin{array}{l}\text { Hemorrhagic } \\
\text { CVA }\end{array}$ & F & 65 & Local clinic \\
\hline & CCF & $\mathrm{M}$ & 81 & $\begin{array}{l}\text { Local } \\
\text { hospital }\end{array}$ \\
\hline \multirow[t]{5}{*}{ September } & $\begin{array}{l}\text { Hypovolemic } \\
\text { shock post } \\
\text { trauma }\end{array}$ & $\mathrm{M}$ & 27 & Local clinic \\
\hline & $\begin{array}{l}\text { Urosepsis with } \\
\text { urinary retention }\end{array}$ & $\mathrm{M}$ & 49 & Local clinic \\
\hline & Severe sepsis & $\mathrm{M}$ & 73 & Local clinic \\
\hline & PCP & $\mathrm{F}$ & 40 & Local clinic \\
\hline & $\begin{array}{l}\text { Acute } \\
\text { abdomen(uterine } \\
\text { perforation) }\end{array}$ & $\mathrm{F}$ & 39 & $\begin{array}{l}\text { Local } \\
\text { hospital }\end{array}$ \\
\hline
\end{tabular}

8 patients died in A/E. $63 \%$ were ranging 14-49 years old. 
Table 2.7. patients died in A/E in October 2014

\begin{tabular}{|c|c|c|c|c|}
\hline & Diseases & Gender & Age & $\begin{array}{l}\text { Sources of } \\
\text { referral }\end{array}$ \\
\hline & $\begin{array}{l}\text { Tension } \\
\text { pneumothorax } \\
\text { post stab wound }\end{array}$ & $\mathrm{M}$ & 39 & Ems \\
\hline & $\begin{array}{l}\text { Severe } \\
\text { dehydration } \\
\text { /gastroenteritis }\end{array}$ & $F$ & 3 months & Local clinic \\
\hline & $\begin{array}{l}\text { Severe } \\
\text { pneumonia }\end{array}$ & $\mathrm{F}$ & 22 & Local clinic \\
\hline \multirow[t]{4}{*}{ October } & $\begin{array}{l}\text { Advanced cancer } \\
\text { of oral cavity }\end{array}$ & M & 69 & Local hospital \\
\hline & Hypoglycemia & $F$ & 41 & Local clinic \\
\hline & $\begin{array}{l}\text { Sepsis/anemia } \\
\text { post infected } \\
\text { bedsores }\end{array}$ & $\mathrm{F}$ & 38 & Local clinic \\
\hline & $\begin{array}{l}\text { Pulmonary } \\
\text { edema/CCF }\end{array}$ & $\mathrm{M}$ & 81 & Local clinic \\
\hline
\end{tabular}

7 patients died during this period. The patient with Tension pneumothorax died due to a delay in management. 1 infant died due to a diarrheal disease and $57 \%$ of patients were middle age. $57 \%$ of dead were female.

Table 2.8. patients died in A/E in November 2014

\begin{tabular}{|l|l|l|l|l|}
\hline & Diseases & Gender & Age & $\begin{array}{l}\text { Sources of } \\
\text { referral }\end{array}$ \\
\hline & PTB & M & 30 & Local clinic \\
\hline November & $\begin{array}{l}\text { Lower GI } \\
\text { bleeding }\end{array}$ & M & 54 & Local clinic \\
\hline & Sepsis & F & 29 & Local clinic \\
\hline & $\begin{array}{l}\text { Hypovolemic } \\
\text { shock }\end{array}$ & F & 66 & $\begin{array}{l}\text { Local } \\
\text { hospital }\end{array}$ \\
\hline $\begin{array}{l}\text { Severe head } \\
\text { injury post RTA }\end{array}$ & M & 8 & $\begin{array}{l}\text { Local } \\
\text { hospital }\end{array}$ \\
\hline & $\begin{array}{l}\text { Metastatic liver } \\
\text { cancer }\end{array}$ & M & 73 & EMS \\
\hline
\end{tabular}

6 deaths recorded. 2 third were female. $50 \%$ of dead were beyond 50 years old and under age died due after being involved into a Road traffic accident.

Table 2.9. patients died in A/E in December 2014

\begin{tabular}{|l|l|l|l|l|}
\hline & $\begin{array}{l}\text { Severe respiratory } \\
\text { syndrome? cause }\end{array}$ & $\mathrm{M}$ & 51 & Local clinic \\
\hline & Hypokalemia & $\mathrm{F}$ & 74 & Self \\
\hline & $\begin{array}{l}\text { Ruptured } \\
\text { esophageal } \\
\text { varicose }\end{array}$ & $\mathrm{M}$ & 23 & Local clinic \\
\hline & Hi post RTA & $\mathrm{F}$ & $8 m o n t h s$ & $\begin{array}{l}\text { Local } \\
\text { hospital }\end{array}$ \\
\hline & $\begin{array}{l}\text { Bilateral subdural } \\
\text { hematoma }\end{array}$ & $\mathrm{M}$ & 59 & $\begin{array}{l}\text { Local } \\
\text { hospital }\end{array}$ \\
\hline Polythrauma post & $\mathrm{M}$ & 23 & Local \\
\hline
\end{tabular}


Texila International Journal of Medicine

Volume 4, Issue 2, Dec 2016

\begin{tabular}{|l|l|l|l|l|}
\hline & RTA & & & hospital \\
\hline & Severe pneumonia & $\mathrm{F}$ & 29 & Local clinic \\
\hline December & $\begin{array}{l}\text { Mouth lower lip } \\
\text { ulcer/abscess }\end{array}$ & $\mathrm{M}$ & 47 & EMS \\
\hline & Cancer blood & $\mathrm{M}$ & 29 & $\begin{array}{l}\text { Local } \\
\text { hospital }\end{array}$ \\
\hline & $\begin{array}{l}\text { Internal bleeding } \\
\text { post RTA }\end{array}$ & $\mathrm{M}$ & 69 & Local clinic \\
\hline
\end{tabular}

10 deaths, upon which 70\% were male and $30 \%$ female including a female infant involved in road traffic accident. $50 \%$ of death was middle age.

Table 2.10. Patients died in A/e in January 2015

\begin{tabular}{|l|l|l|l|l|}
\hline & Diseases & Gender & Age & $\begin{array}{l}\text { Sources of } \\
\text { referral }\end{array}$ \\
\hline & Meningitis & F & 57 & Local clinic \\
\hline & $\begin{array}{l}\text { Intestinal } \\
\text { obstruction }\end{array}$ & F & 59 & $\begin{array}{l}\text { Local } \\
\text { hospital }\end{array}$ \\
\hline January15 & $\begin{array}{l}\text { Severe } \\
\text { dehydration post } \\
\text { GE }\end{array}$ & M & 6 & $\begin{array}{l}\text { Local } \\
\text { hospital }\end{array}$ \\
\hline & Anemia & F & 4 & Local clinic \\
\hline & CCF & M & 71 & Local clinic \\
\hline & Stab wound & M & 25 & EMS \\
\hline & Anorectal sinus & F & 69 & EMS \\
\hline
\end{tabular}

7 patients died including 2 pediatrics patients which have some medical conditions. 57\%were female. And $57 \%$ were beyond 50 years old.

Table 2.11. Patients died in A/E in February 2015

\begin{tabular}{|l|l|l|l|l|}
\hline & Disease & Gender & Age & $\begin{array}{l}\text { Sources of } \\
\text { referral }\end{array}$ \\
\hline & $\begin{array}{l}\text { Head injury } \\
\text { post RTA }\end{array}$ & $\mathrm{M}$ & 46 & EMS \\
\hline $\begin{array}{l}\text { Head injury } \\
\text { post assault }\end{array}$ & $\mathrm{M}$ & 19 & $\begin{array}{l}\text { Local } \\
\text { hospital }\end{array}$ \\
\hline $\begin{array}{l}\text { DKA with } \\
\text { severe } \\
\text { metabolic } \\
\text { acidosis }\end{array}$ & $\mathrm{M}$ & 50 & EMS \\
\hline February & $\begin{array}{l}\text { Sepsis } \\
\text { Acute kidney } \\
\text { injury with } \\
\text { metabolic } \\
\text { acidosis }\end{array}$ & $\mathrm{M}$ & 43 & $\begin{array}{l}\text { Local } \\
\text { hospital }\end{array}$ \\
\hline & PTB & $\mathrm{M}$ & $\begin{array}{l}\text { Local } \\
\text { hospital }\end{array}$ \\
\hline & PCP & $\mathrm{M}$ & 46 & EMS \\
\hline
\end{tabular}

7 deaths. All were male and $85 \%$ of them ranged between 14 and 49 years old. 
Table 2.12. Patients died in A/e in March 2015

\begin{tabular}{|c|c|c|c|c|}
\hline & Disease & Gender & Age & $\begin{array}{l}\text { Source of } \\
\text { referral }\end{array}$ \\
\hline & $\begin{array}{l}\text { End stage renal } \\
\text { failure/HTA/DM }\end{array}$ & $\mathrm{M}$ & 67 & $\begin{array}{l}\text { Local } \\
\text { clinic }\end{array}$ \\
\hline & $\begin{array}{l}\text { Sepsis with hepatic } \\
\text { failure }\end{array}$ & $\mathrm{F}$ & 36 & EMS \\
\hline & $\begin{array}{l}\text { Acute pulmonary } \\
\text { edema }\end{array}$ & $\mathrm{F}$ & 32 & $\begin{array}{l}\text { Local } \\
\text { clinic }\end{array}$ \\
\hline & PCP & $\mathrm{M}$ & 2months & $\begin{array}{l}\text { Local } \\
\text { hospital }\end{array}$ \\
\hline March & Hypokalemia/anemia & $\mathrm{F}$ & 54 & $\begin{array}{l}\text { Local } \\
\text { clinic }\end{array}$ \\
\hline & Bronchiectasis & $\mathrm{F}$ & 52 & $\begin{array}{l}\text { Local } \\
\text { hospital }\end{array}$ \\
\hline & $\begin{array}{l}\text { Urosepsis with } \\
\text { hydronephrosis/bph }\end{array}$ & $\mathrm{M}$ & 85 & EMS \\
\hline & Cancer prostate/copd & $\mathrm{M}$ & 67 & EMS \\
\hline & $\begin{array}{l}\text { Advanced } \\
\text { canceresophagus }\end{array}$ & $\mathrm{F}$ & 66 & $\begin{array}{l}\text { Local } \\
\text { clinic }\end{array}$ \\
\hline & $\begin{array}{l}\text { Polythrauma post } \\
\text { RTA }\end{array}$ & $\mathrm{F}$ & 38 & $\begin{array}{l}\text { Local } \\
\text { hospital }\end{array}$ \\
\hline & $\begin{array}{l}\text { Intracranial } \\
\text { hemorrhage }\end{array}$ & F & 67 & $\begin{array}{l}\text { Local } \\
\text { hospital }\end{array}$ \\
\hline & $\begin{array}{l}\text { Brain mass with ?PE } \\
\text { post DVT/ severe } \\
\text { dehydration }\end{array}$ & $\mathrm{M}$ & 64 & $\begin{array}{l}\text { Local } \\
\text { hospital }\end{array}$ \\
\hline
\end{tabular}

12 patients were recorded death in March 2015 including 1 infant who had severe pneumocystis carinii pneumonia. $67 \%$ had more than 50years old and 58\% were female.

In summary, the previous 12 tables recorded the patients died in Accident and emergency par month. They give us an idea about their ages, genders and the sources of referral. The types of diagnosis are clearly stated. Patients died either from acute or chronic conditions. The next few tables and graphs will expose these deaths with some specificities.

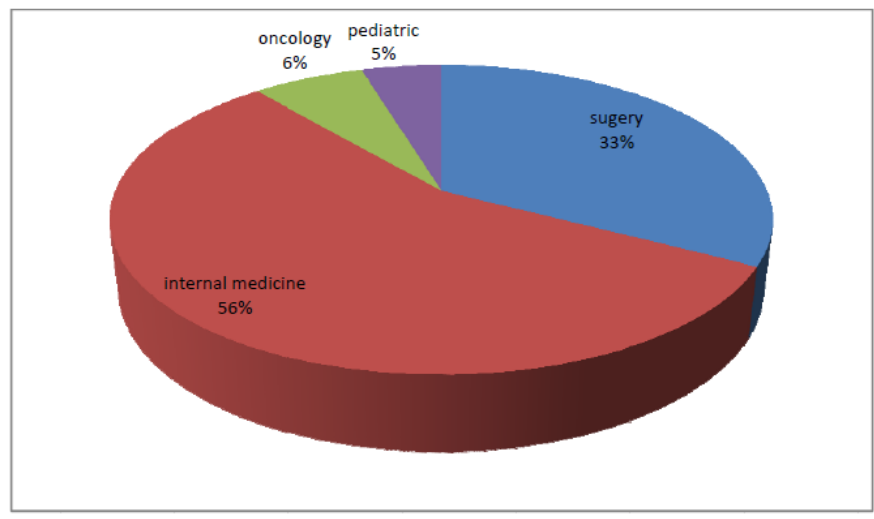

Graph 1. Summary of patients died in A/E by specialty

The above graph shows the patients died based of the specialty of interest. Most patients have an internal medical condition at $56 \%$, 33\% patients had some surgical conditions including traumas. $6 \%$ of patients died from cancer and $5 \%$ was pediatric patients. 
Texila International Journal of Medicine

Volume 4, Issue 2, Dec 2016

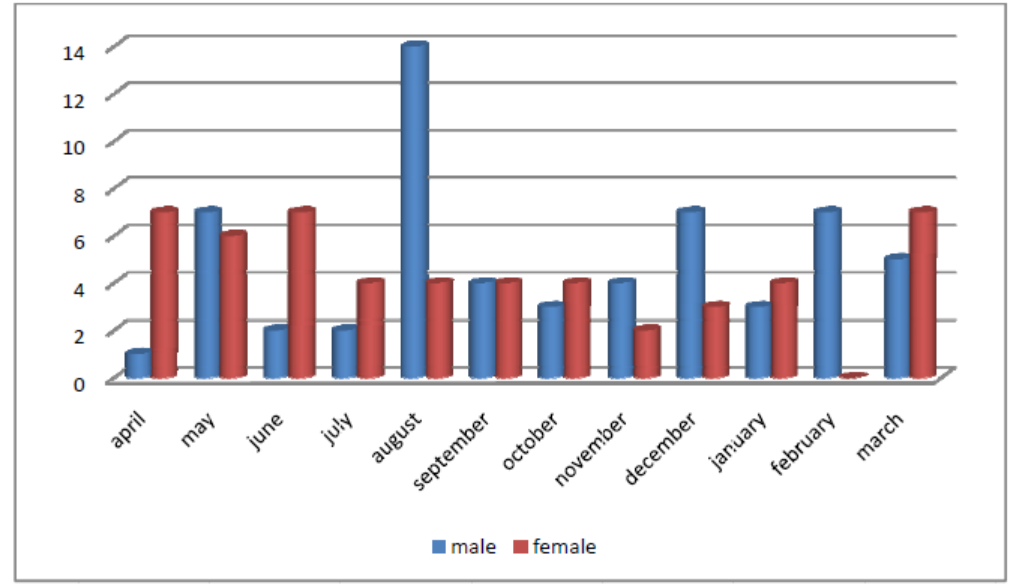

Graph 2. Patients died in A/E by sex

The histogram shows how patients died in a/e were selected per gender. It doesn't show match different in the management of the patients. Overall out of the 111 patients died in $\mathrm{A} / \mathrm{E}$, 59 (53\%) were male and 52 (47\%) were female.

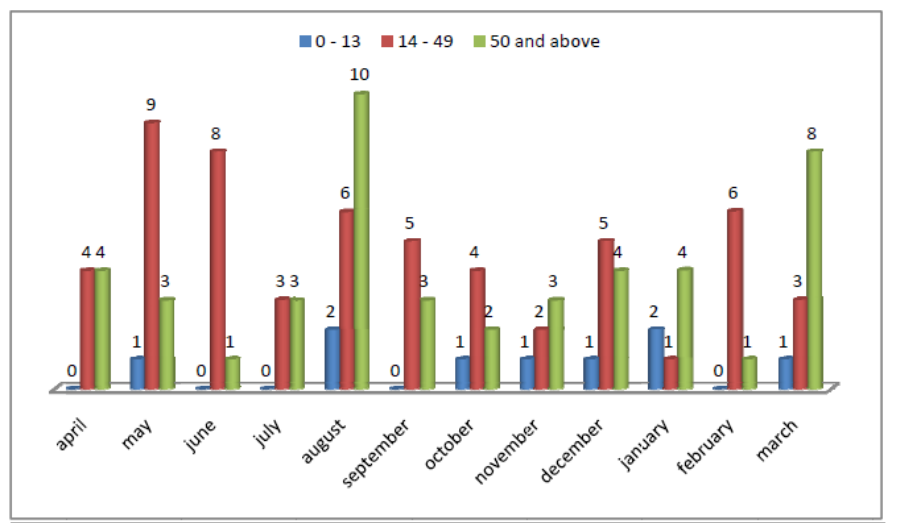

Graph 3. Patients died in A/E by age

Generally, very few pediatric patients died in a/e. young adult and mostly elderly with some chronic disease have shown to be the most patient died in a/e. 9(8\%) children died, $56(51 \%)$ deaths ranged on $13-49$ years and 46(41\%) deaths from 50 years and above.

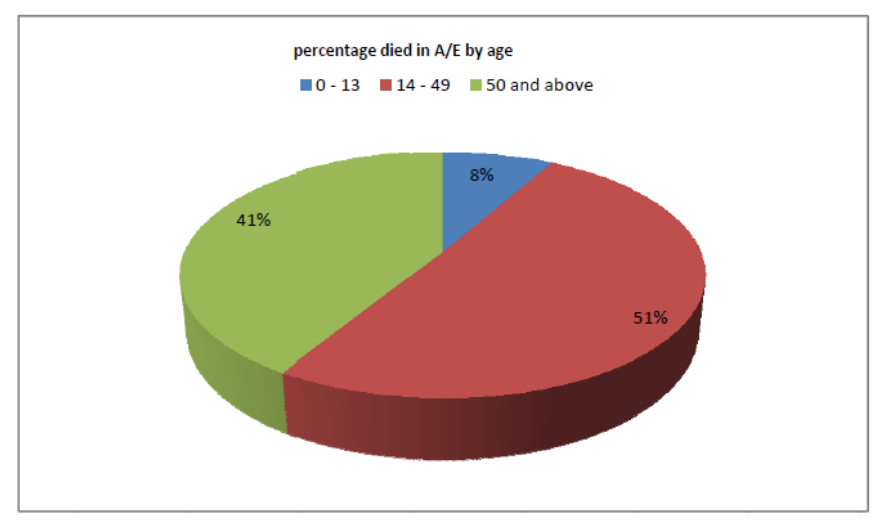

Graph 4. Overall patients died in A/E by age

$51 \%$ of patients died as young adult (14 to 49 ). $41 \%$ were above 50 and pediatric representing $8 \%$. 


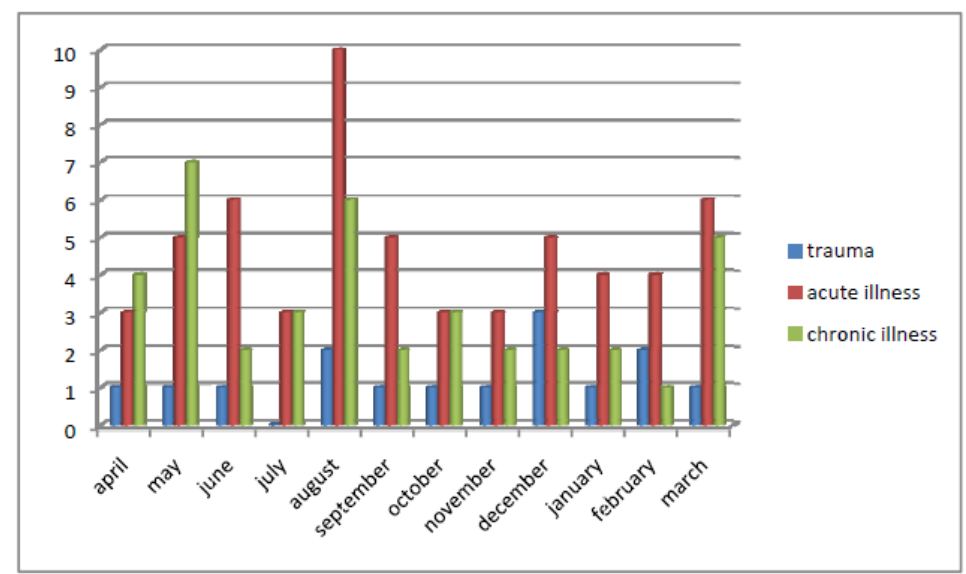

Graph 5. Trauma vs. non traumatic cause of death

The histogram above compares the condition which causes death to some patients each month. In overall, most patients died from acute illness followed by chronic patients. Trauma is not a highest cause of mortality our settings.

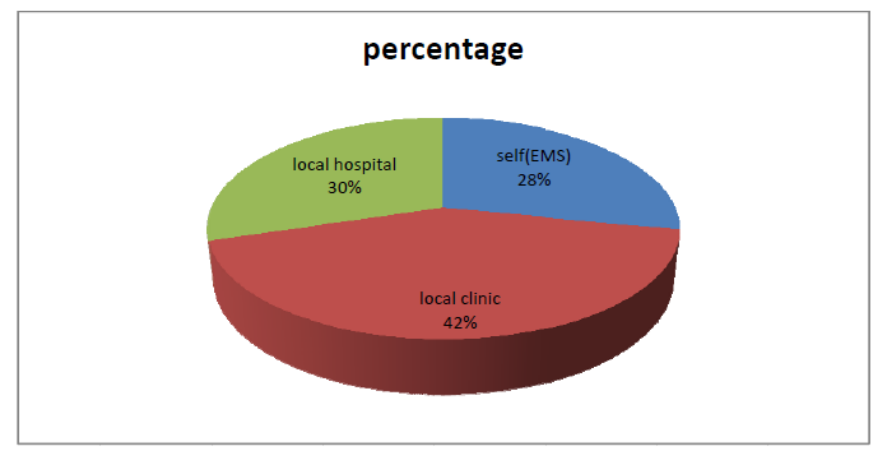

Graph 6. Patients died in A/E per sources of referral

The pi-graph shows the source of referral. $42 \%$ came from local clinic in the same area with the hospital. $30 \%$ from local hospitals while $28 \%$ self-referred.

\section{Case discussion}

We will not close this article without presentation at least on patient died during his stay in our department. Many cases had been reported and discussed during the past meetings due to their interest. Our attention had been retained by a trauma patient presented to the department from local hospital. We are going to report and discuss the issues raised in the management of this patient.

This is a patient M.O., male, 32 year old referred from district hospital with a diagnosis of Polytrauma post road traffic accident( fracture distal Left humerus, Pelvic Left ischio ilio pubic bone, old fracture both tibia/fibula proximal and distal)

The patient arrived in the department of accident and emergency accompanied by a nurse at $01.05 \mathrm{AM}$. The patient was registered at $05.21 \mathrm{AM}$ and attended to by an $\mathrm{A} / \mathrm{E}$ triage nurse 2 minutes later.

At this time, the complaints remain the same with the vital signs as followed: T36.4, RR15, P124, and BP129/93

The patient was codified "orange” as per the triage system used in our setting.

The emergency doctor attended to the patients about 30 minutes later, raising the same complaints like RTA victim who was hit by amoving vehicle and sustained multiple injuries.

The patient was smelling alcohol with blood stained on the clothes. Despite of the pain, the patient was communicating well with a spontaneous breathing. A diagnosis of Polytrauma was made. Some investigations were done and the relevant specialties were informed about the patients. 
Texila International Journal of Medicine

Volume 4, Issue 2, Dec 2016

The general surgeon attended the patient at 9am and planned to review after an abdominal scan is done. The orthopedic medical officer attended the patient at 9.20am and made a diagnosis of multiple fractures. He informed the orthopedic surgeon and prepared the patient for operation.

At 1120, the anesthetist was called. He reported being busy with another patient. He requested that the patient can be prepared for operation.

At 11.30, a new emergency from the surgical ward had been taken to theatre. It was a weekend and there was shortage of staff in theatre.

The patient remains in accident and emergency until when theatre will be free.

At 12.00, the vital sign shows that the patient is in hypovolemic shock.

The patient was called for theatre at 16.05, time when the patient deteriorated.

At 16.07 the 2 surgeons were informed to go to theatre and at 16.15 the patient had a cardiac arrest.

The patient was resuscitated unsuccessfully and was certified death at 17.20.

This case retained our attention due to some multiple issues in management which had been raised.

The patient was a young adult with all his future in front.

Time management and communication were very poor during the follow up of this patient. Despite of the fact that the patient was in shock from arrival, there was a delay in taking the patient to theatre. Also another case from the ward took over a patient with an internal bleeding in accident and emergency. The handling of a hand-over patient: this patient remains in accident and emergency during 3 different shifts. Mostly hand-over patient has a high rate of mortality in accident and emergency worldwide. The shortage of staff and the availability of facilities like theatre room during the weekend should also be considered as risk factors to lose some patients.

\section{Discussion and challenges}

\section{Discussion of result}

During this budgetary year, the accident and emergency department received about 27000 patients. Out of this number, less than 0.5 patients died in the department. This result is equally comparable to the one reported in the 2013 compilation of morbidity and mortality.

Table 2 shows us different diseases which led to death in emergency room. Patients can come in walking or in a stretcher in accident and emergency. Each accident of emergency expects to receive acutely ill patient. But our recorded shows quite significant number of chronically-ill patients. This can be explained by the fact that our setting is the main entrance of the hospital and some chronic patients can have some acute exacerbation of their conditions while other are on end-stage of their disease( e.g. patients with cancers on palliative care).

Table 3 completes the previous one showing the specialty which has the highest number of death. Internal medicine represents almost half of the patient died in the casualty department due partly to the high prevalence of chronically ill condition like HIV/AIDS and cancer as well as some metabolic disorder and cardiovascular disorder.

Looking to the graph 5, generally trauma patients are well managed in our setting. This explains why patients died due to trauma are less in number compare to non- traumatic cause of death (acute and chronic illness together). But an audit in trauma patient is still important. I am refereeing to the case presented previously as an example of a poorly manage patient. But this circumstance was explained by a poor availability of resources.

It was also found that more than half of patients died during that year were between 14 and 49 years old. Considering that chronic diseases have the highest number of death, some questions need to be answered through other researches to try and establish the link between the age and death.

Based on the source of referral, patients were coming from different source. The source of referral should not indicate the severity of the condition. We need to emphasize the 
management of self-referral patients. 28\% patients were self-referred and died in Accident and emergency. this has the same strength of importance with the patient referred from local hospitals (district and primary hospitals). It also means that walk-in patients should receive the same attention as referred patients until proven otherwise.

\section{Challenges}

The management of patients in accident and emergency need skills and teamwork. The challenges will be summarized on

- lack of team work mostly

- Lack of experienced medical and nurse staff

- Shortage of staff

\section{Teamwork:}

To save life especially in a trauma patients need a multidisciplinary approach. Based on the case presented previously, it shows clearly there was miscommunication between teams and specialties.

\section{Experience and inexperienced officers in accident and emergency}

The report shows a pick of death in august. During this period there is movement of medical and nursing staff within the hospital.

\section{Shortage of staff}

In the previous case, the patient was found in cardiac arrest due to the low number of staff in the department.

\section{Conclusion and recommendations}

Accident and emergency at princess marina hospital has remained a center of excellence; and a departmental headlight for the entire hospital as well as the country.

During this report, the accident and emergency shows its capability to contain a high number of patients at a very low rate of death. This is very appreciable for the department. Still some challenges are raised and need to be addressed in goal to reduce the death rate to 0 .

An effort has to be made to help chronically ill patient on the management of their condition. This will improve adherence to treatment and the quality of life for most of them.

Some delays in the management of patients by the admitting team (other specialties) led to dramatic death of some of our patients. This exposed clearly a poor collaboration from other specialty.

Based on the above mentioned findings, we recommend

- Staff training should be provided regularly to improve the quality of care of patient;

- Staff shortage should be resolved as soon as possible;

- Trauma team or multidisciplinary team should be established urgently to allow their continuous presence on the side of the Polytrauma patient;

- Staff should not be transferred in bulk as this reduces the quality of care in the department.

\section{References}

[1] Brennan TA, Leape LL, Laird NM, et al. (1991) Incidence of adverse events and negligence in hospitalized patients: Results of the Harvard Medical Practice Study I. New Engl J Med 324:370-376

[2] "Canadian Institute for Health Information, HSMR: A New Approach for Measuring

[3] Deis JN, Smith KM, Warren MD, Throop PG, Hickson GB, and Joers BJ, et al. (2010) 'Transforming the morbidity and mortality conference into an instrument for system wide improvement’. http://www.ahrq.gov/downloads/pub/ advances2/vol2/Advances-Deis_82.pdf. Accessed May 9, 2010

[4] D.L. Clarcke (2013) "using a structured morbidity and mortality meeting to understand the contribution of human error to adverse surgical events in a south African regional hospital”; 
Texila International Journal of Medicine

Volume 4, Issue 2, Dec 2016

[5] Friedman JN, Pinard MS, Laxer RM. (2005) "The morbidity and mortality conference in university-affiliated pediatric departments in Canada”. J Pediatr; 146:1-2.

[6] Hospital Mortality Trends in Canada (Ottawa: CIHI, 2007)”.

[7] Jed D. Gonzalo, Julius J. Yang, Grace C. Huang (2012) 'Systems-Based Content in Medical Morbidity and Mortality Conferences: A Decade of Change'.

[8] Juliet Higginson and al. (2012) "Mortality and morbidity meetings: an untapped resource for improving the governance of patient safety?” www.patientsafetyfirst.nhs.uk

[9] Kravet SJ, Howell E, Wright SM. (2006)`Morbidity and mortality conference, grand rounds, and the ACGME’s core competencies’. J Gen Intern Med.; 21(11):1192-1194.

[10] 'Making the Medical Morbidity, Mortality, and Improvement Conference Even Better', J Oncol Pract May 1, 2015:e434-e436

[11] Morbidity and Mortality Revisited: Applying a New Quality Improvement Paradigm in Oncology Daniel G. Stover, and Jessica A. Zerillo, 2015

[12] Orlander JD, Fincke BG. (2003) Morbidity and mortality conference: a survey of academic internal medicine departments. J Gen Intern Med; 18:656-8.

[13] Orlander JD, Barber TW, Fincke BG. (2002) 'the morbidity and mortality conference: the delicate nature of learning from error'. Acad Med.; 77(10):1001-1006.

[14] Pierluissi E, Fischer MA, Campbell AR, et al. (2003) Discussion of medical errors in morbidity and mortality conferences. JAMA; 290:2838-42.

[15] Steven J Kravet and Al. (2006) "morbidity and mortality conference, grand rounds, and the ACGME’s core competencies”, J. Gen Med;

[16] Schwarz D, Schwarz R, Gauchan B, et al. (2011) Implementing a systems-oriented morbidity and mortality conference in remote rural Nepal for quality improvement. BMJ Qual Saf; 20:1082-8.

[17] Szostek JH, Wieland ML, Loertscher LL, Nelson DR, Wittich CM, McDonald FS, et al.(2010) ‘ A systems approach to morbidity and mortality conference’. Am J Med. ;123(7):663-668

[18] Wachter RM, Shojania KG, Saint S, et al. (2002) Learning from our mistakes: quality grand rounds, a new case-based series on medical errors and patient safety. Ann Intern Med; 136:850-2.

[19] Wachter RM, Pronovost PJ (2009) Balancing “no blame” with accountability in patient safety. New Engl J Med 361:1401-1406.

[20] Wachter RM, Pronovost PJ (2009) Balancing “no blame” with accountability in patient safety. New Engl J Med 361:1401-1406. 